\title{
Long-term outcomes of follicular variant vs classic papillary thyroid carcinoma
}

\author{
Lauren E Henke', John D Pfeifer ${ }^{2}$, Thomas J Baranski ${ }^{3}$, Todd DeWees ${ }^{4}$ and Perry W Grigsby ${ }^{1,5}$ \\ 'Department of Radiation Oncology, Washington University School of Medicine, St. Louis, Missouri, USA \\ ${ }^{2}$ Department of Pathology, Washington University School of Medicine, St. Louis, Missouri, USA \\ ${ }^{3}$ Division of Endocrinology, Diabetes, and Metabolism, Department of Internal Medicine, Washington University School of Medicine, Saint Louis, \\ Missouri, USA \\ ${ }^{4}$ Division of Biomedical Statistics and Informatics, Mayo Clinic, Scottsdale, Arizona, USA \\ ${ }^{5}$ Division of Nuclear Medicine, Mallinckrodt Institute of Radiology, Washington University School of Medicine, Saint Louis, Missouri, USA \\ Correspondence should be addressed to P W Grigsby: pgrigsby@wustl.edu
}

\begin{abstract}
The majority of papillary thyroid carcinoma (PTC) cases comprise classic papillary (C-PTC) and follicular variant (FV-PTC) histologic sub-types. Historically, clinical equivalency was assumed, but recent data suggest C-PTC may have poorer outcomes. However, large single-institution series with long-term outcomes of C-PTC and FV-PTC, using modern pathologic criteria for FV-PTC, are needed. Our objective was to compare prevalence and impact of clinicopathologic factors, including BRAF mutation status, on long-term outcomes of C-PTC and FV-PTC. We hypothesized that patients with C-PTC would have higher risk disease features and worse survival outcomes. This retrospective study included 1293 patients treated at a single, US academic institution between 1943 and 2009 with mean follow-up of 8.6 years. All patients underwent either partial or total thyroidectomy and had invasive C-PTC or FV-PTC per modern pathology criteria. Primary study measurements included differences in recurrence-free survival (RFS), diseasespecific survival (DSS) and associations with clinicopathologic factors including the BRAF mutation. Compared to FV-PTC, C-PTC was associated with multiple features of highrisk disease $(P<0.05)$ and significantly reduced RFS and DSS. Survival differences were consistent across univariate, multivariate and Kaplan-Meier analyses. BRAF mutations were more common in C-PTC $(P=0.002)$. However, on Kaplan-Meier analysis, mutational status did not significantly impact RFS or DSS for patients with either histologic sub-type. C-PTC therefore indicates higher-risk disease and predicts for significantly poorer longterm outcomes when compared to FV-PTC. The nature of this difference in outcome is not explained by traditional histopathologic findings or by the BRAF mutation.
\end{abstract}

\section{Key Words}

- classic papillary thyroid carcinoma

- follicular variant of papillary thyroid carcinoma

- BRAF mutation

- C-PTC

FV-PTC

\section{Introduction}

Papillary thyroid carcinoma (PTC) is the most common endocrine malignancy, accounting for $88 \%$ of thyroid carcinomas $(1,2)$. Prognosis remains excellent and treatment, typically involving surgery followed by radioactive iodine (RAI), is curative for the majority of PTC patients $(2,3)$. However, up to $10 \%$ of patients experience disease recurrence and as many as 5\% suffer cancer-specific mortality (2). There has been significant historical effort to identify clinical and pathologic markers, such as histologic subtype or somatic mutation profile, which might stratify patients at risk for these poor long-term outcomes.

The majority of PTC cases comprise two histologic subtypes: classic papillary (CP) and follicular variant (FV) https://ec.bioscientifica.com

https://doi.org/10.1530/EC-18-0264 (c) 2018 The authors Published by Bioscientifica Ltd

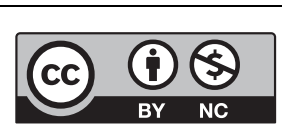

This work is licensed under a Creative Commons Attribution-NonCommercial 4.0 International License. 
PTC, which account for $55-65 \%$ and $23-41 \%$ of cases, respectively $(4,5,6)$. Although several rarer subtypes of PTC (e.g., diffuse sclerosing, tall cell and columnar cell) are established indicators of poorer prognosis, FV-PTC and CP-PTC were historically believed to be low-risk subtypes that were essentially therapeutically and prognostically equivalent $(4,5,7,8,9,10)$. In contrast, some recent studies - including the analysis of 500 PTCs by the Cancer Genome Atlas research group in 2014 - have demonstrated a clear difference in genetic profiles and clinical outcomes between the two, linking C-PTC to increased prevalence of thyroid capsule invasion, LNM, disease recurrence and mortality $(11,12,13)$. However, pathologic classification of FV-PTC has changed significantly since 2016, when non-invasive encapsulated FV-PTC was re-categorized. Specifically, tumors that do not invade the tumor capsule and/or demonstrate vascular invasion (previously grouped as FV-PTC) are now grouped separately as non-invasive follicular thyroid neoplasm with papillary-like nuclear features (NIF-TP), a diagnosis that continues to be refined (14). The most recent large series comparing outcomes between CP-PTC and FV-PTC are from multi-institutional databases without pathology review to evaluate whether patients included had invasive FV-PTC by the new criteria, and thus, their results could be skewed to show falsely favorable outcomes in FV-PTC patients $(10,13,15)$. Given these considerations, a large single-institution study with long follow-up to directly compare clinicopathologic features and outcomes of the two subtypes, with pathology criteria that exclude NIF-TP, is needed.

Similarly, while the prevalence of common somatic mutations has been increasingly investigated in these two sub-types, the comparative impact of these mutations on long-term outcomes and survival of patients with C-PTC vs FV-PTC is not well established (12). The most common and arguably most investigated of these are mutations in the BRAF gene. Occurring in approximately 50\% of PTC patients, the $B R A F$ mutation causes constitutive activation of the MAP-kinase pathway, leading to increased propensity for tumor cell proliferation $(16,17)$. Although the prognostic value of the $B R A F$ mutation in PTC at large is controversial, its role in the differential long-term outcomes of FV-PTC vs C-PTC has not been specifically investigated in a large series and merits further analysis $(18,19,20,21,22)$. Notably, BRAF mutations have been shown to occur less often in FV-PTC than in C-PTC and could possibly play distinct roles in the outcomes of patients with one histologic subtype vs the other $(12,18$, 23, 24).

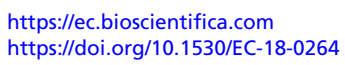

This study analyzes a cohort of 1293 patients with either FV-PTC or C-PTC treated at Washington University School of Medicine between 1943 and 2009, a subset of which were tested for the BRAF mutation. To our knowledge, this is the largest single-institution study to date of outcomes in C-PTC vs FV-PTC, using pathology criteria that exclude NIF-TP and is one of few to address and compare the role of $B R A F$ mutations in these two subtypes. The goal of this retrospective study was to compare the prevalence and impact of clinicopathologic factors, including $B R A F$ mutational status, on long-term outcomes of FV-PTC vs C-PTC.

\section{Materials and methods}

\section{Patient identification and clinicopathologic} data collection

This retrospective study was approved by the Human Research Protection Office at Washington University School of Medicine, including retrospective chart review (protocol number 201010705) with waiver of consent. Records of 1712 patients with invasive thyroid cancer who were referred to the Department of Radiation Oncology at Washington University between 1943 and 2009 were queried. The data set was interrogated for patients with thyroid carcinoma of follicular cell origin who met the following criteria: underwent either partial or total thyroidectomy, received follow-up care at Washington University in St. Louis, and had C-PTC or FV-PTC. A total of 1293 patients met criteria. Thyroid tumor specimens were available for a subset of 542 patients and were obtained from an archived bank of formalinfixed, paraffin-embedded (FFPE) thyroid tissue. Tumor histologies of anaplastic, non-invasive, undifferentiated and NIF-TP were excluded. Data abstracted from patient records included histological subtype, treatment records and clinicopathologic outcomes. Review of records indicated that none of these individuals had any history of therapeutic radiation exposure. $B R A F$ mutational status was determined after surgical and medical treatments of all patients were concluded and did not affect treatment decisions.

\section{PTC histological and sub-type classification}

Hematoxylin and eosin-stained slides were examined for all cases by Washington University School of Medicine pathologists to diagnose histologic subtype

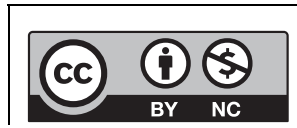

This work is licensed under a Creative Commons Attribution-NonCommercial 4.0 International License. 
or to confirm outside hospital diagnoses. Diagnoses of classic papillary subtype of PTC were made using standard criteria, by identification of areas with classic characteristics of PTC, including papillary architecture, typical PTC nuclei (enlarged, overlapping, irregular, ground-glass empty nuclei with nuclear grooves), psammoma bodies and stromal reaction (25). Follicular variant subtype of PTC was identified by standard criteria of the same classic PTC cellular and nuclear characteristics in the setting of follicular architecture (25, $26,27,28)$. At our institution, $>95 \%$ of a tumor specimen must demonstrate FV-PTC histology to be classified as such. Cases of NIF-TP were excluded. This pathologic distinction is reliable in this study despite our inclusion of historic patients because our institution historically classified FV-PTC tumors that were fully or partially encapsulated or encapsulated with vascular invasion separately from invasive FV-PTCs, and this subset of cases, which includes NIF-TP cases, was excluded (12). While time of diagnosis of some included referral cases of FV-PTC predated its recognition as a distinct histologic sub-type, all pathology diagnoses were made in subsequent years by Washington University faculty pathologists, using standard diagnostic criteria. Histologically, the 1293 cases included here comprised 956 cases of C-PTC and 337 cases of FV-PTC.

\section{BRAF mutation subset analysis}

For a subset of patients, $B R A F$ mutational status was also determined at our institution. For 542 patients, surgically removed thyroid tumor specimens were available and identified from within the Thyroid Cancer Tumor Registry and obtained from an archived bank of formalin-fixed, paraffin-embedded (FFPE) thyroid tissue. For all cases identified, hematoxylin and eosin-stained slides were re-examined by study pathologists to confirm histologic subtype, meaning that original pathologic diagnoses were specifically reviewed and re-confirmed for nearly half $(542 / 1293)$ of the total study cohort. None of these cases were re-classified. Areas of carcinoma were marked by study pathologists on the glass slides to guide collection of tissue cores from the corresponding FFPE tumor blocks of the case.

Two tissue cores of $1 \mathrm{~mm}$ diameter were extracted from the areas of tumor by means of disposable biopsy punches with plungers (Miltex, York, PA). Samples were incubated in xylene for three minutes at $50^{\circ} \mathrm{C}$; xylene aspiration was followed by two washes with $100 \%$ ethanol. Subsequently, samples were incubated for $48 \mathrm{~h}$ with $0.5 \mathrm{mg} / \mathrm{mL}$ proteinase $\mathrm{K}$ (Qiagen), with a mid-interval addition of $0.5 \mathrm{mg} / \mathrm{mL}$ proteinase $\mathrm{K}$. DNA was extracted from each sample via a commercial kit (Puregene, Minneapolis, MN, USA) according to the manufacturer's instructions. Following extraction, DNA was stored at $4^{\circ} \mathrm{C}$. Polymerase chain reaction (PCR) was utilized to amplify the 215 base pair (bp) BRAF exon 15, using previously published primers and Platinum Taq DNA Polymerase (Invitrogen), as has been described in the literature $(29,30)$. Samples were then subjected to restriction fragment length polymorphism (RFLP) analysis by the enzyme TspRI (Invitrogen), using the buffer conditions recommended by the manufacturer. TspRI cuts the wild-type, $215 \mathrm{bp}$ amplification product into two fragments of 120 and $95 \mathrm{bp}$. After the restriction digest, the DNA bands were resolved by agarose gel electrophoresis and visualized by ethidium bromide staining. RFLP analysis has been used by numerous other labs to evaluate BRAF exon 15 and we have extensively validated the assay for use in our own laboratory, as previously reported $(29,30,31)$.

\section{Treatment and follow-up}

The vast majority of patients underwent total thyroidectomy (93\%). Cervical lymphadenectomy was performed in $57 \%$ of patients. Postoperative ${ }^{131} \mathrm{I}$ was administered to $90 \%$ of patients. The administered activity of ${ }^{131}$ I given as initial treatment was determined based on our general treatment guidelines $(32,33)$. Our standard adult treatment recommendations during the study years included an administered activity of 100 millicuries (mCi) of ${ }^{131} \mathrm{I}$ for patients without nodal involvement, while lymph node-positive patients received $150 \mathrm{mCi}$. Patients with distant metastatic disease typically received $250 \mathrm{mCi}$, with a median total ${ }^{131} \mathrm{I}$ administration of $400 \mathrm{mCi}$ (range 0-1400, mean $435 \mathrm{mCi}$ ); ${ }^{131} \mathrm{I}$ administrations were repeated for patients with persistently positive wholebody ${ }^{131}$ I scintigraphy. Median values of initial and cumulative administered activity of ${ }^{131} \mathrm{I}$ are reported in Table 1. Surveillance consisted of physical examination and laboratory studies, including thyroid-stimulating hormone (TSH), triiodothyronine and free thyroxine for all patients, with the addition of thyroglobulin levels in the latter years of the study.

\section{Statistical analysis}

Clinical and pathological outcomes and BRAF mutational status were analyzed for significant associations. $P$ values

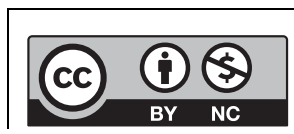

This work is licensed under a Creative Commons Attribution-NonCommercial 4.0 International License. 
Table 1 Patient, tumor and treatment characteristics stratified by histologic sub-type of PTC.

\begin{tabular}{|c|c|}
\hline Characteristic & Number $(\%)$ or Median (range) \\
\hline All patients & $1293(100)$ \\
\hline \multicolumn{2}{|l|}{ Gender } \\
\hline Male & $313(24.2)$ \\
\hline Female & $980(75.8)$ \\
\hline Age at diagnosis (years) & $42.4(4.2-86.2)$ \\
\hline \multicolumn{2}{|l|}{ Race } \\
\hline White & $1120(86.6)$ \\
\hline Black & $106(8.2)$ \\
\hline Asian & $53(4.1)$ \\
\hline Hispanic & $14(1.1)$ \\
\hline \multicolumn{2}{|l|}{ Pathological features } \\
\hline Capsular invasion & $612(47.7)$ \\
\hline Soft tissue invasion & 358 (27.9) \\
\hline Vascular invasion & $190(14.8)$ \\
\hline Positive margins & $344(26.8)$ \\
\hline Tumor size $(\mathrm{cm})$ & $1.5(0.1-13.0)$ \\
\hline Multifocal & $561(43.7)$ \\
\hline Cervical LN involvement & $586(45.8)$ \\
\hline \multicolumn{2}{|l|}{ Extent of disease } \\
\hline Thyroid only & $700(54.2)$ \\
\hline Thyroid and cervical LN & 548 (42.4) \\
\hline Lung metastases & $38(3.4)$ \\
\hline Bone metastases & $5(0.4)$ \\
\hline Brain metastases & $1(0.1)$ \\
\hline \multicolumn{2}{|l|}{ AJCC tumor stage } \\
\hline $\mathrm{T} 1$ & $552(43.3)$ \\
\hline $\mathrm{T} 2$ & $345(27.1)$ \\
\hline T3 & $113(8.9)$ \\
\hline T4 & $264(20.7)$ \\
\hline \multicolumn{2}{|l|}{ AJCC nodal stage } \\
\hline No & $692(54.3)$ \\
\hline N1a & $427(33.5)$ \\
\hline N1b & $156(12.2)$ \\
\hline$B R A F$ mutation positive & 349 (64.4) \\
\hline Initial I-131 dose (mCi) & $150(0-1400)$ \\
\hline Total I-131 dose (mCi) & $150(0-1400)$ \\
\hline
\end{tabular}

$<0.05$ were considered statistically significant and all $P$ values were two-tailed. $T$ tests were used for comparison of data with continuous variables, while chi-squared tests were used for dichotomous data. All variables associated with the BRAF mutation, recurrence-free survival (RFS) or disease-specific survival (DSS) at the $P<0.05$ level were entered into multivariate logistic regression models for $B R A F$ positivity, decreased RFS and decreased DSS. To remove redundancy and improve the predictive value of the multivariate analysis, complex variables such as AJCC stage were reduced to the variables they comprise, such as histologic tumor size, extrathyroidal extension and location of disease at diagnosis. Kaplan-Meier analysis was also performed to estimate the RFS and DSS probabilities for $B R A F$ mutation positive vs negative patient groups. Statistical analyses were performed using SAS, version 9.2 (SAS Institute).

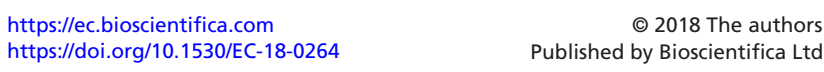

\begin{tabular}{|c|c|}
\hline Follicular variant PTC $(\%)$ & $P$ value \\
\hline $337(26.1)$ & $\begin{array}{c}\text { NA } \\
0.118\end{array}$ \\
\hline \multicolumn{2}{|l|}{$71(21.1)$} \\
\hline \multicolumn{2}{|l|}{266 (78.9) } \\
\hline $44.2(8.3-84.6)$ & $\begin{array}{l}0.435 \\
0.005\end{array}$ \\
\hline \multicolumn{2}{|l|}{$280(83.1)$} \\
\hline \multicolumn{2}{|l|}{$42(12.5)$} \\
\hline \multicolumn{2}{|l|}{$10(3.0)$} \\
\hline \multicolumn{2}{|l|}{$5(1.5)$} \\
\hline $124(36.8)$ & $<0.001$ \\
\hline $64(19.0)$ & $<0.001$ \\
\hline $55(16.3)$ & 0.367 \\
\hline \multirow[t]{2}{*}{$73(21.7)$} & 0.013 \\
\hline & $<0.001$ \\
\hline 132 (39.3) & 0.058 \\
\hline \multirow[t]{2}{*}{$102(30.5)$} & $<0.001$ \\
\hline & $<0.001$ \\
\hline $236(70.0)$ & $<0.001$ \\
\hline $92(27.3)$ & $<0.001$ \\
\hline $9(2.7)$ & 0.735 \\
\hline $0(0)$ & 0.184 \\
\hline \multirow[t]{2}{*}{$0(0)$} & 0.482 \\
\hline & $<0.001$ \\
\hline \multicolumn{2}{|l|}{134 (40.5) } \\
\hline \multicolumn{2}{|l|}{109 (32.9) } \\
\hline \multicolumn{2}{|l|}{$43(13.0)$} \\
\hline \multicolumn{2}{|l|}{$45(13.6)$} \\
\hline & $<0.001$ \\
\hline \multicolumn{2}{|l|}{$230(69.3)$} \\
\hline \multicolumn{2}{|l|}{$83(25.0)$} \\
\hline \multicolumn{2}{|l|}{$19(5.7)$} \\
\hline $73(53.3)$ & 0.002 \\
\hline $130(0-750)$ & 0.998 \\
\hline $150(0-902.8)$ & 0.185 \\
\hline
\end{tabular}

\section{Results}

\section{Patient characteristics}

A total of 1293 patients meeting eligibility criteria were included in the study. Of these, a subset of 542 patients with evaluable tissue underwent $B R A F$ gene analysis. Patient and tumor characteristics are summarized in Table 1. Average age at diagnosis was 43 years (range 4.2-86.2 years, median 42.4 years) and mean follow-up time was 8.6 years (range $0-50.2$ years, median 6.4 years). Review of pathologic features is included in Table 1. In the majority of patients, disease was limited to the thyroid only $(54 \%, 700 / 1292)$ and was unilateral within the thyroid (68\%). However, $42 \%$ also had disease in cervical lymph nodes, while 3\% had lung metastases, $0.4 \%$ had bone metastases and one patient $(0.1 \%)$ had brain metastases. 


\section{Histologic sub-type analysis}

Histologically, 74\% of cases were classified as C-PTC, while FV-PTC comprised 26\%. Initial dose of RAI and type of surgery did not significantly differ between C-PTC vs FV-PTC patient groups $(P=0.998$ and $P=0.265$, respectively). Average follow-up time was 9.5 years for patients with FV-PTC and 10.6 years for patients with C-PTC (median 6.8 and 8.3 years, respectively, $P=0.007$ ). C-PTC histology was associated with greater incidence of thyroid capsule invasion $(P<0.001)$, soft tissue invasion $(P<0.001)$, positive surgical margins $(P=0.013)$ and cervical LNM $(P \leq 0.001)$. Compared to patients with FV-PTC, C-PTC patients had increased extent of disease at diagnosis $(P<0.001)$, but interestingly, had smaller histologic tumor size $(P<0.001)$ (Table 1$)$. On multivariate analysis, thyroid capsule invasion (HR 1.82, 95\% CI 1.37 to 2.42 ) was independently predictive of C-PTC. In contrast, disease confined to the thyroid (HR 0.41, 95\% CI 0.31 to 0.55 ) and larger histologic tumor size (HR 0.77, 95\% CI 0.71 to 0.84 ) was predictive of FV-PTC.

\section{$B R A F$ analysis}

On subset analysis of 542 patients, $64.4 \%$ of patients (349/542) had the BRAF mutation. Mutation status was identified in these patients by RFLP, which displayed two, wild-type DNA bands (120 and $95 \mathrm{bp}$ ) digested by the TspRI restriction enzyme when the BRAF V600 mutation was absent, and three DNA bands (215, 120 and $95 \mathrm{bp}$ ) when the mutation was present (heterozygote) (30). BRAF mutations were significantly more likely to be present in patients with C-PTC than in patients with FV-PTC $(P=0.002)$. However, on subset multivariate analysis of 542 patients with established $B R A F$ status, presence of the $B R A F$ mutation did not independently predict C-PTC histology.

\section{Patient outcomes}

For the entire cohort, overall survival was 92.5 and $90.3 \%$ at 10 and 15 years, respectively. RFS at 10 years and 15 years was 81 and $77 \%$, respectively. During the study period, 90 patients died, with 44 of these deaths $(3.4 \%$ of all patients) attributable to PTC. At 10 and 15 years, DSS was 96.5 and $95.5 \%$, respectively. The probability of having recurrent disease was significantly higher for patients with C-PTC than for patients with FV-PTC $(P<0.001)$ (Fig. 1). Similarly, patients with C-PTC were at increased risk of cancer-specific death $(P=0.008)$ (Fig. 2).

$\begin{array}{lr}\text { https://ec.bioscientifica.com } & \text { ○ } 2018 \text { The authors } \\ \text { https://doi.org/10.1530/EC-18-0264 } & \text { Published by Bioscientifica Ltd }\end{array}$

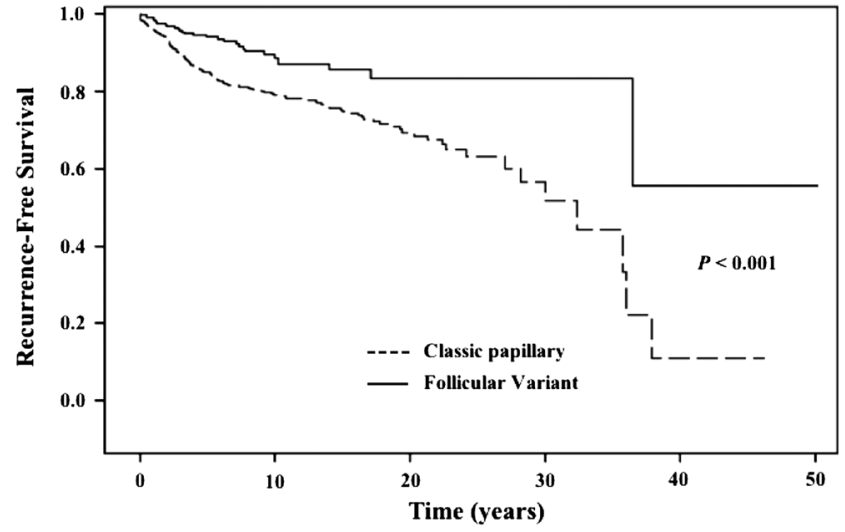

Figure 1

Recurrence-free survival based on histologic sub-type of papillary thyroid carcinoma.

For all-comers, presence of the BRAF mutation was not significantly associated with increased risk of disease recurrence or cancer mortality on univariate or multivariate analyses (Table 2). This was consistent with Kaplan-Meier analysis, which demonstrated no difference in probability of RFS or DSS based on presence of absence of the BRAF mutation $(P=0.594$ and $P=0.182$, respectively). As previously stated, the $B R A F$ mutation was more common in C-PTC than FV-PTC (Table 1). To assess BRAF mutation impact on RFS and DSS probabilities within each histologic sub-type, subset Kaplan-Meier analyses were performed for C-PTC and FV-PTC patient groups. For patients with C-PTC, presence or absence of the $B R A F$ mutation did not significantly impact the probability of disease recurrence or cancer-related death ( $P=0.766$ and $P=0.096$, respectively). Similarly, $B R A F$ status did not impact recurrence or survival for patients with FV-PTC ( $P=0.906$ and $P=0.313$, respectively).

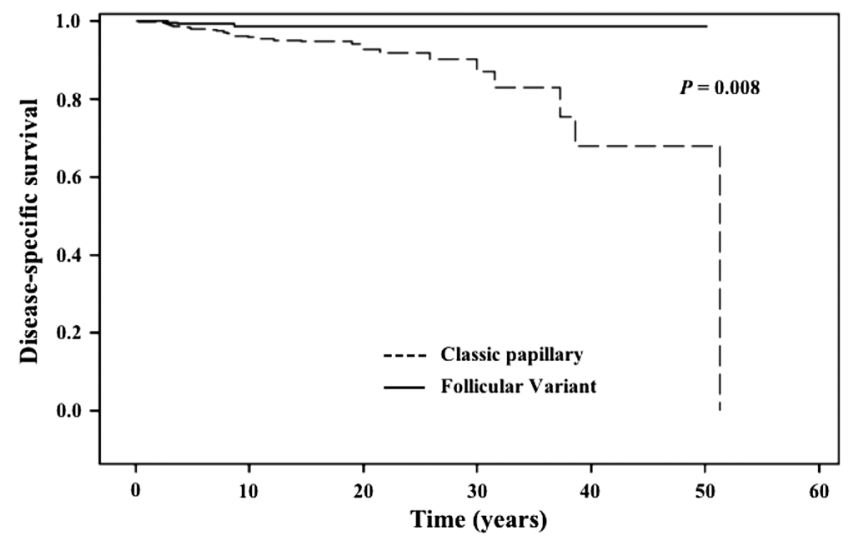

Figure 2

Disease-specific survival based on histologic sub-type of papillary thyroid carcinoma. 


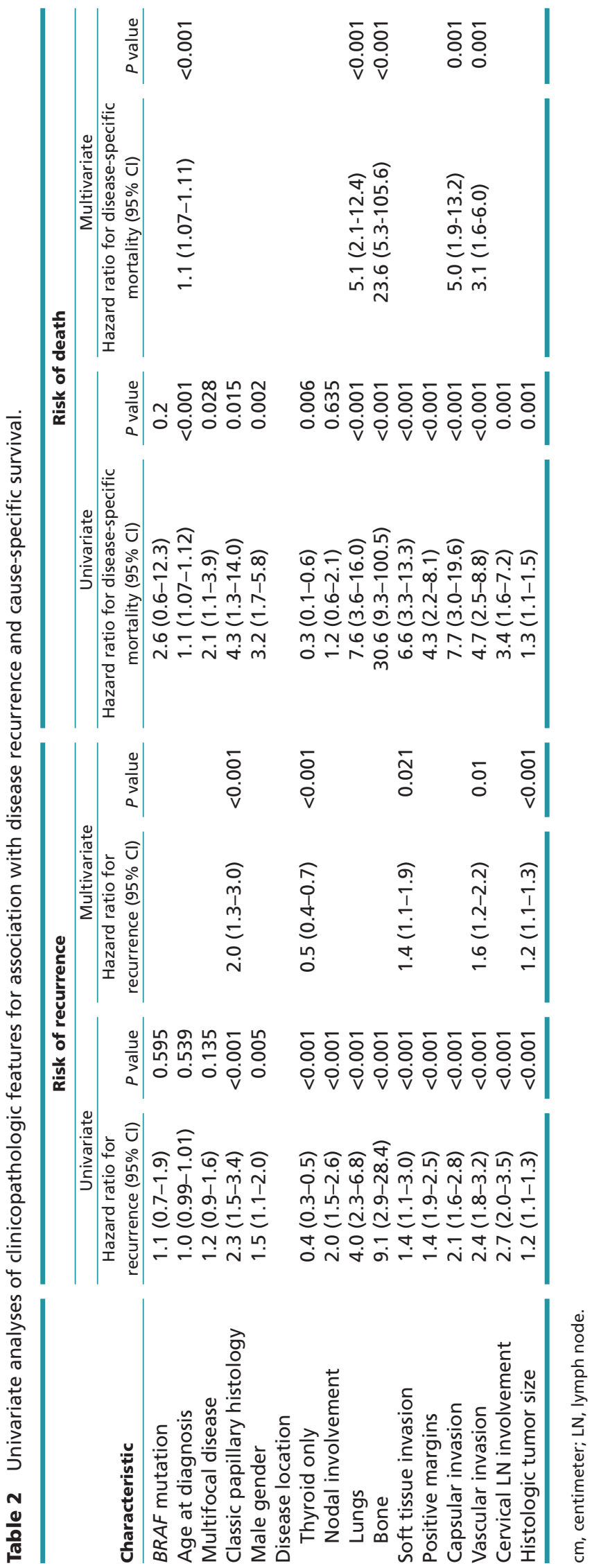

https://ec.bioscientifica.com https://doi.org/10.1530/EC-18-0264
() 2018 The authors Published by Bioscientifica Ltd
Apart from histologic sub-type, multiple established clinicopathologic characteristics of high-risk disease were correlated with increased recurrence and disease-specific mortality (Table 2). Of these, microscopic tumor size, vascular invasion and soft tissue invasion independently predicted recurrence on multivariate analysis, while disease confined to the thyroid was protective. Independent predictors of disease-specific mortality included vascular invasion, thyroid capsule invasion, increased patient age and distant metastases to the lungs or bones at the time of diagnosis (Table 2).

\section{Discussion}

The primary goal of this study was to compare the clinicopathologic characteristics and prevalence of the $B R A F$ mutation in C-PTC and FV-PTC and their relationship to long-term patient outcome, using more modern pathology criteria for FV-PTC. Historic series comparing the two most common histologic sub-types of PTC failed to demonstrate clear therapeutic or prognostic differences between the two, while recent studies have indicated that C-PTC portends a poorer prognosis than FV-PTC. In this large analysis, we found consistent evidence that C-PTC is associated with numerous clinicopathologic risk factors, in addition to increased prevalence of the BRAF mutation. Although the BRAF mutation was more common in patients with C-PTC, it was not an independent predictor of high-risk disease. Instead, we found that C-PTC itself was a strong predictor of poorer outcomes, including significantly increased risk of recurrence and diseasespecific mortality, even though we excluded cases of encapsulated or partially encapsulated tumors or those encapsulated with vascular invasion, such as NIF-TP. This was uniformly observed across univariate, multivariate and Kaplan-Meier analyses.

A majority of patients in our study had C-PTC (74\%), while FV-PTC was less common (26\%), which is consistent with the literature $(4,5,6)$. There was no difference in patient management (using initial RAI dose as a proxy) between the two histologic sub-types of patients. Although follow-up time was slightly longer for patients with C-PTC vs FV-PTC (Table 1), mean follow-up for each group was extensive (10.6 and 9.4 years, respectively). In PTC, nearly all disease recurrences and the large majority of cancerspecific deaths occur less than one decade after diagnosis $(34,35)$. Mean follow-up time for each of the patient groups in our analysis met this time frame. Differences in outcomes observed between the two patient groups are

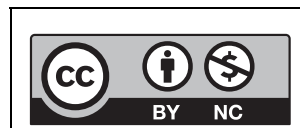

This work is licensed under a Creative Commons Attribution-NonCommercial 4.0 International License. 
therefore highly unlikely to be attributable to difference in follow-up time.

This analysis included comparison of the associations between known clinicopathologic factors of poor prognosis and histologic sub-types of PTC. On univariate analysis, C-PTC was associated with numerous clinicopathologic features of high-risk disease, including thyroid capsule invasion, soft tissue invasion, vascular invasion, positive surgical margins, LNM and advanced AJCC nodal stage (Table 1). Of these, thyroid capsule invasion was independently predictive of C-PTC histology on multivariate analysis. Our findings are consistent with the recent series by Cheng et al. in 2011, where C-PTC was associated with increased risk of LNM, thyroid capsule invasion and vascular invasion. Additionally, several small prior studies have also shown that C-PTC is associated with increased local invasion and LNM (36, 37). However, ours is the largest single institution study to date to demonstrate such a clear association between C-PTC and nearly every examined clinicopathologic characteristic of high-risk disease.

In contrast with C-PTC, the only pathologic risk factor significantly associated with FV-PTC was increased microscopic primary tumor size, which remained significant on multivariate analysis (HR 0.77, 95\% CI 0.71 to 0.84 ) and has been previously reported (6). Although FV-PTC predicted for larger tumor size, it also predicted for disease that remained confined to the thyroid at the time of diagnosis (HR 0.41, 95\% CI 0.31 to 0.55), while C-PTC was associated with increased risk for lymph node metastases at the time of diagnosis (Table 1). This supports the growing body of evidence that C-PTC is a biologically unique and more aggressive tumor with more metastatic potential than FV-PTC. Such an interpretation is supported by the recent and extensive TCGA analysis of PTC subtypes, which revealed that C-PTC and FV-PTC differ on the basis of principle driving somatic mutations and cellular signaling, leading to a tendency toward poorer differentiation and aggressive behavior within the C-PTC phenotype (12).

Additionally, we evaluated the relationships between common clinicopathologic features, RFS and DSS. The strongest predictor of increased risk on multivariate analysis for recurrence was classic papillary histology, followed by vascular invasion, soft tissue invasion and histologic tumor size. Apart from C-PTC histology, these are well-documented risk factors and are largely accounted for in current AJCC staging guidelines.(23, 32, 33, 38, 39) For disease-specific mortality, the strongest predictors were distant metastases to the bone and lungs, then extrathyroidal extension, vascular invasion and increased age at diagnosis. These findings are congruent with existing literature $(21,38,39,40)$.

Kaplan-Meier analysis was also performed to further characterize the relationship between histological subtype, RFS and DSS. This demonstrated C-PTC patients not only had significantly increased risk for disease recurrence (Fig. 1), but also had increased risk of cancer-specific death (Fig. 2). The contrast in outcomes between the two histologies is striking. At 20 and 30 years of follow-up, the probability of RFS for FV-PTC patients was stable at approximately $85 \%$, compared with only 70 and 50\% for C-PTC patients (Fig. 1). Similarly, DSS probability at 20 and 30 years was stable at nearly $100 \%$ for FV-PTC patients, compared with only 90 and $85 \%$ for C-PTC patients (Fig. 2). Although a small study of 160 patients with PTC by Burningham et al. in 2005 also noted a statistically significant increase in risk of recurrence for patients with C-PTC, most previous reports have not uncovered a difference in long-term outcomes between C-PTC and FV-PTC $(4,6,8,36,37)$. The most probable cause for the contrast between our findings and the literature is that prior studies suffered small patient cohorts and were underpowered. Our much larger patient cohort, in addition to extensive follow-up time, likely enabled us to expose these significant differences in long-term outcome based on histology.

We also performed a subset analysis of 542 patients to evaluate the relationship between the BRAF mutation, histologic sub-type of PTC and long-term outcomes. Overall, the prognostic value of the BRAF mutation in PTC remains uncertain, but some authors report that it confers increased risk of disease and cancer mortality $(20,21,39,41)$. While our focus in this study was not on the prognostic utility of the BRAF mutation, this analysis is among the largest to specifically characterize its relationship with histologic sub-types of PTC. As has been reported numerous times in the literature, C-PTC histology was significantly associated with presence of the $B R A F$ mutation compared to the FV-PTC on univariate analysis $(11,12,18,23,24,42)$. However, our subset multivariate analysis, demonstrated that BRAF status was not independently predictive of C-PTC histology, or associated with risk of disease recurrence or diseasespecific mortality on univariate or multivariate analysis. These findings are consistent with the findings of Cheng et al., who similarly reported that the BRAF mutation lacked predictive value for high-risk disease features (11). Importantly, our subset Kaplan-Meier analysis also revealed that within each histologic subtype, $B R A F$ is not

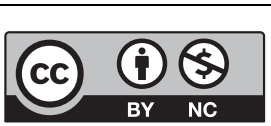

This work is licensed under a Creative Commons Attribution-NonCommercial 4.0 International License. 
a significant predictor of recurrence or death from thyroid cancer. This indicates that the differences we observed in recurrence and survival between C-PTC and FV-PTC are independent of the BRAF mutation.

The greatest strengths of this analysis are our exclusion of NIF-TP within our pathology criteria for our FV-PTC cohort, our large single-institution patient population, and our extended follow-up time (mean 8.6 years, range 50.2 years, median 6.4 years). We are the first to our knowledge to address differences between C-PTC and FV-PTC while excluding NIF-TP. This is a critical point, as other series comparing long-term outcomes in these two either predate the diagnosis of NIF-TP or are multiinstitutional and/or national database studies that cannot provide specific pathology criteria $(10,13,15)$. Without exclusion of NIF-TP, outcomes in FV-PTC could be falsely skewed toward a comparatively improved prognosis, given the benign course of such non-invasive disease. Although a criticism might be that be that our exclusion of all cases of encapsulated or partially encapsulated tumors or those encapsulated with vascular invasion was overly broad, and excluded more than just NIF-TP cases, this in fact strengthens our findings. We have shown that even when one selects more pathologically aggressive FV-PTC tumors for comparison with C-PTC, FV-PTC remains a more favorable diagnosis. Our patient cohort is also approximately three-fold larger than any previous study addressing differences between C-PTC and FV-PTC and has extended follow-up time. Disease recurrence and disease-specific deaths are rare and occur late in PTC, highlighting the importance of extensive follow-up and a large cohort $(34,35)$. One weakness of this analysis is its retrospective nature; most studies of PTC are either retrospective or meta-analyses, given the relative rarity of the disease. In this study, all patients were treated homogenously, according to guidelines, at a single, academic institution. This, combined with our effort to emphasize multivariate analyses, minimizes the likelihood of confounding variables. Our subset analysis of the BRAF mutation included 542/1293 patients, which might also be criticized. Had we determined BRAF status for a larger percent of patients, it is possible that we could have strengthened our analysis. Our reported subset analysis here does remain one of the largest reported single-institution US studies of the BRAF mutation in PTC to date (18).

Overall, this analysis demonstrates that the C-PTC indicates higher-risk disease and predicts for significantly poorer long-term outcomes, including increased risk for disease recurrence and cancer-specific death, when compared to FV-PTC using pathology criteria that exclude NIF-TP. Our study is the largest, single-institution series to date to illustrate that the historical assumption that C-PTC and FV-PTC are clinically equivalent is incorrect. Our findings lend strong support to recent data indicating that C-PTC confers a worse prognosis that was historically overlooked. The nature of this difference in outcome is not explained by traditional histopathologic findings like tumor size or soft tissue invasion or by the $B R A F$ mutation alone. Additionally, our results support the recent findings of the TCGA analysis and the conclusion that C-PTC and FV-PTC are biologically two distinct disease entities with different oncogenic drivers and tumor behaviors. Finally, in light of the increased risk for poor outcomes that patients with C-PTC face compared to their counterparts with FV-PTC, we propose that C-PTC histology be considered a characteristic of higher-risk disease in initial patient evaluation, similar to risk factors such as thyroid capsule invasion and soft tissue invasion. Alteration of disease classification and management to address this risk might be considered, but further studies are also warranted to expand upon our findings.

\section{Declaration of interest}

The authors declare that there is no conflict of interest that could be perceived as prejudicing the impartiality of the research reported.

\section{Funding}

This publication was supported by the Washington University Institute of Clinical and Translational Sciences grant UL1T2000448 from the National Center for Advancing Translational Sciences (NCATS). The content is solely the responsibility of the authors and does not necessarily represent the official views of the $\mathrm{NIH}$.

\section{References}

1 Davies L \& Welch HG. Increasing incidence of thyroid cancer in the United States, 1973-2002. JAMA 2006295 2164-2167. (https://doi. org/10.1001/jama.295.18.2164)

2 Hundahl SA, Fleming ID, Fremgen AM \& Menck HR. A National Cancer Data Base report on 53,856 cases of thyroid carcinoma treated in the U.S., 1985-1995. Cancer 199883 2638-2648. (https:// doi.org/10.1002/(SICI)1097-0142(19981215)83:12<2638::AIDCNCR31>3.0.CO;2-1)

3 Yip L, Nikiforova MN, Carty SE, Yim JH, Stang MT, Tublin MJ, Lebeau SO, Hodak SP, Ogilvie JB \& Nikiforov YE. Optimizing surgical treatment of papillary thyroid carcinoma associated with BRAF mutation. Surgery 2009146 1215-1223. (https://doi.org/10.1016/j. surg.2009.09.011)

4 Carcangiu ML, Zampi G, Pupi A, Castagnoli A \& Rosai J. Papillary carcinoma of the thyroid. A clinicopathologic study of 241 cases treated at the University of Florence, Italy. Cancer $1985 \mathbf{5 5}$ 805-828. (https://doi.org/10.1002/1097-0142(19850215)55:4<805::AIDCNCR2820550419>3.0.CO;2-Z) 
5 Sebastian SO, Gonzalez JM, Paricio PP, Perez JS, Flores DP, Madrona AP, Romero PR \& Tebar FJ. Papillary thyroid carcinoma: prognostic index for survival including the histological variety. Archives of Surgery 2000135 272-277. (https://doi.org/10.1001/ archsurg.135.3.272)

6 Burningham AR, Krishnan J, Davidson BJ, Ringel MD \& Burman KD. Papillary and follicular variant of papillary carcinoma of the thyroid: initial presentation and response to therapy. Otolaryngology - Head and Neck Surgery 2005132 840-844. (https://doi.org/10.1016/j. otohns.2005.02.002)

7 Mazzaferri EL \& Kloos RT. Clinical review 128: current approaches to primary therapy for papillary and follicular thyroid cancer. Journal of Clinical Endocrinology and Metabolism 200186 1447-1463. (https:// doi.org/10.1210/jcem.86.4.7407)

8 Passler C, Prager G, Scheuba C, Niederle BE, Kaserer K, Zettinig G \& Niederle B. Follicular variant of papillary thyroid carcinoma: a longterm follow-up. Archives of Surgery 2003138 1362-1366. (https://doi. org/10.1001/archsurg.138.12.1362)

9 Rosai J, Zampi G \& Carcangiu ML. Papillary carcinoma of the thyroid. A discussion of its several morphologic expressions, with particular emphasis on the follicular variant. American Journal of Surgical Pathology 19837 809-817. (https://doi. org/10.1097/00000478-198307080-00010)

10 Yu X-M, Schneider DF, Leverson G, Chen H \& Sippel RS. Follicular variant of papillary thyroid carcinoma is a unique clinical entity: a population-based study of 10,740 cases. Thyroid 201323 1263-1268. (https://doi.org/10.1089/thy.2012.0453)

11 Cheng S, Serra S, Mercado M, Ezzat S \& Asa SL. A high-throughput proteomic approach provides distinct signatures for thyroid cancer behavior. Clinical Cancer Research 201117 2385-2394. (https://doi. org/10.1158/1078-0432.CCR-10-2837)

12 Cancer Genome Atlas Research Network. Integrated genomic characterization of papillary thyroid carcinoma. Cell 2014159 676-690. (https://doi.org/10.1016/j.cell.2014.09.050)

13 Yang J, Gong Y, Yan S, Shi Q, Zhu J, Li Z, Chen Q \& Gong R. Comparison of the clinicopathological behavior of the follicular variant of papillary thyroid carcinoma and classical papillary thyroid carcinoma: a systematic review and meta-analysis. Molecular and Clinical Oncology 20153 753-764. (https://doi.org/10.3892/mco.2015.540)

14 Nikiforov YE, Seethala RR, Tallini G, Baloch ZW, Basolo F, Thompson LDR, Barletta JA, Wenig BM, Ghuzlan Al A, Kakudo K, et al. Nomenclature revision for encapsulated follicular variant of papillary thyroid carcinoma: a paradigm shift to reduce overtreatment of indolent tumors. JAMA Oncology 20162 1023-1029. (https://doi.org/10.1001/jamaoncol.2016.0386)

15 Shi X, Liu R, Basolo F, Giannini R, Shen X, Teng D, Guan H, Shan Z, Teng W, Musholt TJ, et al. Differential clinicopathological risk and prognosis of major papillary thyroid cancer variants. Journal of Clinical Endocrinology and Metabolism 2016101 264-274. (https://doi. org/10.1210/jc.2015-2917)

16 Xing M. BRAF mutation in thyroid cancer. Endocrine-Related Cancer 200512 245-262. (https://doi.org/10.1677/erc.1.0978)

17 Fagin JA \& Mitsiades N. Molecular pathology of thyroid cancer: diagnostic and clinical implications. Best Practice and Research: Clinical Endocrinology and Metabolism 200822 955-969. (https://doi. org/10.1016/j.beem.2008.09.017)

18 Gouveia C, Can NT, Bostrom A, Grenert JP, van Zante A \& Orloff LA Lack of association of BRAF mutation with negative prognostic indicators in papillary thyroid carcinoma: the University of California, San Francisco, Experience. JAMA Otolaryngology - Head and Neck Surgery 2013139 1164-1170. (https://doi.org/10.1001/ jamaoto.2013.4501)

19 Xing M, Alzahrani AS, Carson KA, Viola D, Elisei R, Bendlova B, Yip L, Mian C, Vianello F, Tuttle RM, et al. Association between BRAF V600E mutation and mortality in patients with papillary thyroid cancer. JAMA 2013309 1493-1501. (https://doi.org/10.1001/ jama.2013.3190)

20 Elisei R, Viola D, Torregrossa L, Giannini R, Romei C, Ugolini C, Molinaro E, Agate L, Biagini A, Lupi C, et al. The BRAFV600E mutation is an independent, poor prognostic factor for the outcome of patients with low-risk intrathyroid papillary thyroid carcinoma: single-institution results from a large cohort study. Journal of Clinical Endocrinology and Metabolism 201297 4390-4398. (https://doi. org/10.1210/jc.2012-1775)

21 Ito Y, Yoshida H, Maruo R, Morita S, Takano T, Hirokawa M, Yabuta T, Fukushima M, Inoue $\mathrm{H}$, Tomoda $\mathrm{C}$, et al. BRAF mutation in papillary thyroid carcinoma in a Japanese population: its lack of correlation with high-risk clinicopathological features and disease-free survival of patients. Endocrine Journal 200956 89-97. (https://doi. org/10.1507/endocrj.K08E-208)

22 Brzeziańska E, Pastuszak-Lewandoska D, Wojciechowska K, Migdalska-Sek M, Cyniak-Magierska A, Nawrot E \& Lewiński A. Investigation of V600E BRAF mutation in papillary thyroid carcinoma in the Polish population. Neuro Endocrinology Letters 2007 28 351-359.

23 Lupi C, Giannini R, Ugolini C, Proietti A, Berti P, Minuto M, Materazzi G, Elisei R, Santoro M, Miccoli P, et al. Association of BRAF V600E mutation with poor clinicopathological outcomes in 500 consecutive cases of papillary thyroid carcinoma. Journal of Clinical Endocrinology and Metabolism 200792 4085-4090. (https://doi. org/10.1210/jc.2007-1179)

24 Fugazzola L, Mannavola D, Cirello V, Vannucchi G, Muzza M, Vicentini L \& Beck-Peccoz P. BRAF mutations in an Italian cohort of thyroid cancers. Clinical Endocrinology 200461 239-243. (https://doi. org/10.1111/j.1365-2265.2004.02089.x)

25 DeLellis RA. Pathology and Genetics of Tumours of Endocrine Organs. Lyon: IARC, 2004.

26 Halperin EC, Brady LW, Perez CA \& Wazer DE. Perez \& Brady's Principles and Practice of Radiation Oncology. Philadephia, PA USA:Lippincott Williams \& Wilkins, 2013.

27 Furlan JC, Bedard YC \& Rosen IB. Role of fine-needle aspiration biopsy and frozen section in the management of papillary thyroid carcinoma subtypes. World Journal of Surgery 200428 880-885. (https://doi.org/10.1007/s00268-004-6953-z)

28 Kurian EM, Dawlett M, Wang J, Gong Y \& Guo M. The triage efficacy of fine needle aspiration biopsy for follicular variant of papillary thyroid carcinoma using the Bethesda reporting guidelines. Diagnostic Cytopathology 201140 E69-E73. (https://doi.org/10.1002/ dc.21718)

29 Zatelli MC, Trasforini G, Leoni S, Frigato G, Buratto M, Tagliati F, Rossi R, Cavazzini L, Roti E \& Degli Uberti EC. BRAF V600E mutation analysis increases diagnostic accuracy for papillary thyroid carcinoma in fine-needle aspiration biopsies. European Journal of Endocrinology 2009161 467-473. (https://doi.org/10.1530/EJE-09-0353)

30 Henke LE, Perkins SM, Pfeifer JD, Ma C, Chen Y, DeWees T \& Grigsby PW. BRAF V600E mutational status in pediatric thyroid cancer. Pediatric Blood and Cancer 201461 1168-1172. (https://doi. org/10.1002/pbc.24935)

31 Lee X, Gao M, Ji Y, Yu Y, Feng Y, Li Y, Zhang Y, Cheng W \& Zhao W. Analysis of differential BRAF(V600E) mutational status in high aggressive papillary thyroid microcarcinoma. Annals of Surgical Oncology 200916 240-245. (https://doi.org/10.1245/s10434-0080233-3)

32 American Thyroid Association (ATA) Guidelines Taskforce on Thyroid Nodules and Differentiated Thyroid Cancer, Cooper DS, Doherty GM, Haugen BR, Kloos RT, Lee SL, Mandel SJ, Mazzaferri EL, McIver B, Pacini F, Schlumberger M, et al. Revised American Thyroid Association management guidelines for patients with thyroid nodules and differentiated thyroid cancer. Thyroid 200919 1167-1214. (https://doi.org/10.1089/thy.2009.0110)

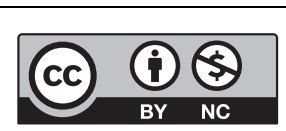

This work is licensed under a Creative Commons Attribution-NonCommercial 4.0 International License. 
33 Cooper DS, Doherty GM, Haugen BR, Kloos RT, Lee SL, Mandel SJ, Mazzaferri EL, McIver B, Sherman SI \& Tuttle RM. Management guidelines for patients with thyroid nodules and differentiated thyroid cancer. Thyroid 200616 109-142. (https://doi.org/10.1089/ thy.2006.16.ft-1)

34 Mazzaferri EL \& Jhiang SM. Differentiated thyroid cancer long-term impact of initial therapy. Transactions of the American Clinical and Climatological Association 1995106 151-170.

35 Durante C, Montesano T, Torlontano M, Attard M, Monzani F, Tumino S, Costante G, Meringolo D, Bruno R, Trulli F, et al. Papillary thyroid cancer: time course of recurrences during postsurgery surveillance. Journal of Clinical Endocrinology and Metabolism 201398 636-642. (https://doi.org/10.1210/jc.2012-3401)

36 Tielens ET, Sherman SI, Hruban RH \& Ladenson PW. Follicular variant of papillary thyroid carcinoma. A clinicopathologic study. Cancer 199473 424-431. (https://doi.org/10.1002/10970142(19940115)73:2<424::AID-CNCR2820730230>3.0.CO;2-I)

37 Zidan J, Karen D, Stein M, Rosenblatt E, Basher W \& Kuten A. Pure versus follicular variant of papillary thyroid carcinoma: clinical features, prognostic factors, treatment, and survival. Cancer 200397 1181-1185. (https://doi.org/10.1002/cncr.11175)

38 Elisei R, Ugolini C, Viola D, Lupi C, Biagini A, Giannini R, Romei C, Miccoli P, Pinchera A \& Basolo F. BRAFV600E mutation and outcome of patients with papillary thyroid carcinoma: a 15-year median follow-up study. Journal of Clinical Endocrinology and Metabolism 200893 3943-3949. (https://doi.org/10.1210/ jc.2008-0607)

39 Xing M, Westra WH, Tufano RP, Cohen Y, Rosenbaum E, Rhoden KJ, Carson KA, Vasko V, Larin A, Tallini G, et al. BRAF mutation predicts a poorer clinical prognosis for papillary thyroid cancer. Journal of Clinical Endocrinology and Metabolism 200590 6373-6379. (https:// doi.org/10.1210/jc.2005-0987)

40 Mazzaferri EL. What is the optimal initial treatment of low-risk papillary thyroid cancer (and why is it controversial)? Oncology 2009 23 579-588.

41 Kebebew E, Weng J, Bauer J, Ranvier G, Clark OH, Duh Q-Y Shibru D, Bastian B \& Griffin A. The prevalence and prognostic value of BRAF mutation in thyroid cancer. Annals of Surgery 2007246 466-470; discussion 470-1. (https://doi.org/10.1097/ SLA.0b013e318148563d)

42 Ricarte-Filho J, Ganly I, Rivera M, Katabi N, Fu W, Shaha A, Tuttle RM, Fagin JA \& Ghossein R. Papillary thyroid carcinomas with cervical lymph node metastases can be stratified into clinically relevant prognostic categories using oncogenic BRAF, the number of nodal metastases, and extra-nodal extension. Thyroid 201222 575-584. (https://doi.org/10.1089/thy.2011.0431)

Received in final form 4 October 2018

Accepted 8 October 2018

Accepted Preprint published online 9 October 2018
This work is licensed under a Creative Commons Attribution-NonCommercial 4.0 International License. 